\title{
A general decay and optimal decay result in a heat system with a viscoelastic term
}

\author{
Abderrahmane Youkana( ${ }^{(1)}$, Salim A. Messaoudi ${ }^{(2)}$ \& Aissa Guesmia ${ }^{(3)}$ \\ (1) Department of Mathematics, University of Batna 2 \\ Batna 05078, Algeria \\ E-mail: abderrahmane.youkana@univ-batna2.dz \\ abder.youkana@yahoo.fr \\ (2) Department of Mathematics and Statistics \\ KFUPM, Dhahran 31261 \\ Saudi Arabia \\ E-mail: messaoud@kfupm.edu.sa \\ (3) Elie Cartan Institute of Lorraine, Bat. A \\ Lorraine - Metz University, Ile de Saulcy, 57045 \\ Metz Cedex 01, France \\ E-mail: aissa.guesmia@univ-lorraine.fr
}

\begin{abstract}
We consider a quasilinear heat system in the presence of an integral term and establish a general and optimal decay result from which improves and generalizes several stability results in the literature.
\end{abstract}

\section{Introduction}

In this work, we consider the following problem:

$$
\begin{cases}A(t)\left|u_{t}\right|^{m-2} u_{t}-\Delta u+\int_{0}^{t} g(t-s) \Delta u(x, s) d s=0 & \text { in } \Omega \times(0,+\infty), \\ u(x, t)=0 & \text { in } \partial \Omega \times \mathbb{R}^{+}, \\ u(x, 0)=u_{0}(x) & \text { in } \Omega,\end{cases}
$$

where $m \geq 2, \Omega$ is a bounded domain of $\mathbb{R}^{n}, n \in \mathbb{N}^{*}$, with a smooth boundary $\partial \Omega$, $g: \mathbb{R}^{+} \rightarrow \mathbb{R}^{+}$is a positive nonincreasing function, and $A: \mathbb{R}^{+} \rightarrow M_{n}(\mathbb{R})$ is a bounded square matrix satisfying $A \in C\left(\mathbb{R}^{+}\right)$and, for some positive constant $c_{0}$,

$$
(A(t) v, v) \geq c_{0}|v|^{2}, \quad \forall t \in \mathbb{R}^{+}, \forall v \in \mathbb{R}^{n},
$$

where (.,.) and |.| are the inner product and the norm, respectively, in $\mathbb{R}^{n}$. The equation in consideration arises from various mathematical models in engineering 
and physics. For instance, in the study of heat conduction in materials with memory, the classical Fourier law is replaced by the following form (cf. [9]):

$$
q=-d \nabla u-\int_{-\infty}^{t} \nabla(k(x, t) u(x, \tau)) d \tau
$$

where $u$ is the temperature, $d$ the diffusion coefficient and the integral term represents the memory effect in the material. This type of problems has considered by a number of researchers; see $[2,9,11]$ and the references therein. From a mathematical point of view, we expect that the integral term would be dominated by the leading term in the equation, so that the theory of parabolic equation can be applied. In fact, this has been confirmed by the work of Yin [11], in which he considered a general equation of the form

$$
u_{t}=\operatorname{div} A\left(x, t, u, u_{x}\right)+a\left(x, t, u, u_{x}\right)+\int_{0}^{t} \operatorname{div} B\left(x, t, \tau, u, u_{x}\right) d \tau
$$

and proved the existence of a unique weak solution under suitable conditions on $A$, $B$ and $a$. See more results concerning global existence and asymptotic behavior in Nakao and Ohara [7], Nakao and Chen [8], and Engler et al. [3]. Pucci and Serrin [10] discussed the following system:

$$
A(t)\left|u_{t}\right|^{m-2} u_{t}=\Delta u-f(x, u),
$$

for $m>1$ and $f$ satisfying

$$
(f(x, u), u) \geq 0
$$

and showed that strong solutions tend to the rest state as $t \rightarrow+\infty$, however, no rate of decay has been given. Berrimi and Messaoudi [1] showed that, if $A$ satisfies (1.2), then solutions with small initial energy decay exponentially for $m=2$ and polynomially if $m>2$. Messaoudi and Tellab [5] considered (1.1), under condition (1.2) and for relaxation function $g$ satisfying a general decay condition of the form

$$
g^{\prime}(t) \leq-\xi(t) g(t), \quad \forall t \in \mathbb{R}^{+},
$$

for some nonincreasing differentiable function $\xi: \mathbb{R}^{+} \rightarrow \mathbb{R}^{+}$, and established a general decay result, from which the exponential and polynomial decay rates of [1] are only special cases. Recently, Liu and Chen [4] investigated (1.1), with a nonlinear source term, and established a general decay result under suitable conditions on $g$ and the nonlinear source term. They also proved a blow-up result for the solution with both positive and negative initial energy.

In this work, we discuss (1.1) when $g$ is of a more general decay, and establish a general and optimal decay result, which improves those of Berrimi and Messaoudi [1], Liu and Chen [4], and Messaoudi and Tellab [5]. 


\section{Preliminaries}

In this section, we present some material needed in the proof of our result. For the relaxation function $g$ we assume that

$\left(G_{1}\right)$ The function $g: \mathbb{R}^{+} \rightarrow \mathbb{R}^{+}$is a differentiable function satisfying

$$
g(0)>0 \quad \text { and } \quad 1-\int_{0}^{+\infty} g(s) d s=l>0 .
$$

$\left(G_{2}\right)$ There exist a constant $p \in[1,3 / 2)$ and a nonincreasing differentiable function $\xi: \mathbb{R}^{+} \rightarrow \mathbb{R}^{+}$satisfying

$$
g^{\prime}(t) \leq-\xi(t) g^{p}(t), \quad \forall t \in \mathbb{R}^{+}
$$

$\left(G_{3}\right)$ We also assume that

$$
\begin{array}{ll}
2 \leq m \leq \frac{2 n}{n-2} & \text { if } n \geq 3 \\
m \geq 2 & \text { if } n=1,2 .
\end{array}
$$

Remark 2.1. There are many functions satisfying (G1) and (G2). Examples of such functions are, for $b>0, \alpha>0, \nu>1$, and $a>0$ small enough,

$$
g_{1}(t)=a e^{-b(t+1)^{\alpha}} \quad \text { and } \quad g_{2}(t)=\frac{a}{(1+t)^{\nu}} .
$$

We will also be using the embedding $H_{0}^{1}(\Omega) \hookrightarrow L^{q}(\Omega), L^{r}(\Omega) \hookrightarrow L^{q}(\Omega)$, for $2 \leq q \leq r<+\infty$, and Poincaré's inequality. The same embedding constant $C_{*}$ will be used, and $C$ denotes a generic positive constant.

We introduce the following:

$$
E(t)=\frac{1}{2}(g \circ \nabla u)(t)+\frac{1}{2}\left(1-\int_{0}^{t} g(s) d s\right)\|\nabla u(t)\|_{2}^{2}, \quad \forall t \in \mathbb{R}^{+}
$$

where $\|\cdot\|_{q}=\|\cdot\|_{\left(L^{q}(\Omega)\right)^{n}}$, for $1 \leq q<+\infty$, and

$$
(g \circ \nabla u)(t)=\int_{0}^{t} g(t-\tau)\|\nabla u(., t)-\nabla u(., \tau)\|_{2}^{2} d \tau, \quad \forall t \in \mathbb{R}^{+} .
$$

Similarly to [10], we give the definition of a strong solution of (1.1).

Definition 2.1. A weak solution of $(1.1)$ on $[0, T]$ is a function

$$
u \in C\left([0, T) ;\left(H_{0}^{1}(\Omega)\right)^{n}\right) \cap C^{1}\left((0, T) ;\left(L^{m}(\Omega)\right)^{n}\right)
$$


which satisfies

$$
\begin{gathered}
\int_{0}^{t} \int_{\Omega}\left(\nabla u(x, s)-\int_{0}^{s} \nabla u(x, \tau) d \tau\right) \cdot \nabla \phi(x, s) d x d s \\
\quad+\int_{0}^{t} A(s)\left|u_{t}\right|^{m-2} u_{t}(x, s) \cdot \phi(x, s) d x d s=0
\end{gathered}
$$

for all $t$ in $[0, T)$ and all $\phi$ in $C\left([0, T) ;\left(H_{0}^{1}(\Omega)\right)^{n}\right)$.

Remark 2.2. Similarly to [10], we assume the existence of a solution. For the linear case $(m=2)$, one can easily establish the existence of a weak solution by the Galerkin method. In the one-dimensional case $(n=1)$, the existence is established in a more general setting by Yin [11].

Finally, we state an important lemma [6].

Lemma 2.1. Assume that $g$ satisfies (G1) and (G2) and $u$ is the solution of (1.1), then there exists a positive constant $k_{0}$ such that

$$
\xi(t)(g \circ \nabla u)(t) \leq k_{0}\left(-E^{\prime}(t)\right)^{\frac{1}{2 p-1}}, \quad \forall t \in \mathbb{R}^{+} .
$$

We also recall the following particular case of the well-known Jensen inequality which will be of essential use in obtaining our result: let $f: \Omega \rightarrow \mathbb{R}^{+}$and $h: \Omega \rightarrow \mathbb{R}^{+}$ be integrable functions on $\Omega$ such that

$$
\int_{\Omega} h(x) d x=k>0
$$

Then, for any $p>1$, we have

$$
\frac{1}{k} \int_{\Omega}(f(x))^{\frac{1}{p}} h(x) d x \leq\left(\frac{1}{k} \int_{\Omega} f(x) h(x) d x\right)^{\frac{1}{p}} .
$$

\section{Decay result}

In this section, we state and prove our main result. We start with a lemma.

Lemma 3.1. Let $u$ be the solution of (1.1). Then the energy satisfies

$$
E^{\prime}(t)=-\int_{\Omega} A(t)\left|u_{t}\right|^{m} d x-\frac{1}{2} g(t)\|\nabla u(t)\|_{2}^{2}+\frac{1}{2}\left(g^{\prime} \circ \nabla u\right)(t) \leq 0, \quad \forall t \in \mathbb{R}^{+} .
$$

Proof. By multiplying the first equation in (1.1) by $u_{t}$, integrating over $\Omega$ we get (3.1), after routine manipulations. 
Lemma 3.2. Let $u$ be a solution of problem (1.1). Then, for any $\delta>0$, we have

$$
\|\nabla u(t)\|_{2}^{2} \leq c_{4} \delta E(t)-\frac{C_{\delta}}{c_{0}} E^{\prime}(t)+c_{5}(g \circ \nabla u)(t), \quad \forall t \in \mathbb{R}^{+},
$$

where $c_{0}$ is introduced in $(1.2), c_{4}$ and $c_{5}$ are two positive constants, and $C_{\delta}$ is a positive constant depending on $\delta$.

Proof. Multiplying the first equation in (1.1) by $u$ and integrating over $\Omega$, we get

$$
\|\nabla u(t)\|_{2}^{2}=-\int_{\Omega} A(t)\left|u_{t}\right|^{m-2} u_{t} u(x, t) d x+\int_{\Omega} \int_{0}^{t} g(t-s) \nabla u(x, s) \cdot \nabla u(x, t) d s d x .
$$

Now, we estimate the right-hand side of (3.3). By using Young's and Poincaré's inequalities, the boundedness of $A$, conditions $\left(G_{1}\right)$ and $\left(G_{3}\right)$, and the fact that

$$
E(t) \leq E(0)
$$

we find, for any $\delta>0$,

$$
\begin{aligned}
-\int_{\Omega} A(t)\left|u_{t}\right|^{m-2} u_{t} u d x & \leq \delta\|u(., t)\|_{m}^{m}+C_{\delta}\left\|u_{t}(., t)\right\|_{m}^{m} \\
& \leq \delta C_{*}^{m}\|\nabla u(., t)\|_{2}^{m}+C_{\delta}\left\|u_{t}(., t)\right\|_{m}^{m} \\
& \leq \delta C_{*}^{m}\left(\frac{2 E(0)}{l}\right)^{\frac{m-2}{2}}\left(\frac{2}{l} E(t)\right)+C_{\delta}\left\|u_{t}(., t)\right\|_{m}^{m} \\
& \leq c_{1} \delta E(t)-\frac{C_{\delta}}{c_{0}} E^{\prime}(t) .
\end{aligned}
$$

Next, we estimate the second term of the right-hand side of (3.3) carefully. By Young's inequality, we easily see that

$$
\begin{aligned}
& \int_{\Omega} \nabla u(x, t) \cdot \int_{0}^{t} g(t-s) \nabla u(x, s) d s d x \leq \frac{1}{2}\|\nabla u(., t)\|_{2}^{2} \\
+ & \frac{1}{2} \int_{\Omega}\left(\int_{0}^{t} g(t-s)(|\nabla u(x, s)-\nabla u(x, t)|+|\nabla u(x, t)|) d s\right)^{2} d x .
\end{aligned}
$$

Using the fact that

$$
\int_{0}^{t} g(s) d s \leq 1-l
$$

and Young's and Hölder's inequalities, we obtain, for any $\eta>0$,

$$
\begin{aligned}
& \int_{\Omega}\left(\int_{0}^{t} g(t-s)(|\nabla u(x, s)-\nabla u(x, t)|+|\nabla u(x, t)|) d s\right)^{2} d x \\
= & \int_{\Omega}\left(\int_{0}^{t} g(t-s)(|\nabla u(s)-\nabla u(t)|)\right)^{2} d x+\int_{\Omega}\left(\int_{0}^{t} g(t-s)|\nabla u(t)| d s\right)^{2} d x \\
+ & 2 \int_{\Omega}\left(\int_{0}^{t} g(t-s)(|\nabla u(s)-\nabla u(t)|) d s\right) \quad\left(\int_{0}^{t} g(t-s)|\nabla u(t)| d s\right) d x
\end{aligned}
$$




$$
\begin{aligned}
& \leq(1+\eta) \int_{\Omega}\left(\int_{0}^{t} g(t-s)|\nabla u(t)| d s\right)^{2} d x+\left(1+\frac{1}{\eta}\right) \int_{\Omega}\left(\int_{0}^{t} g(t-s)|\nabla u(s)-\nabla u(t)| d s\right)^{2} d x \\
& \leq(1+\eta)(1-l)^{2}\|\nabla u(., t)\|_{2}^{2}+\left(1+\frac{1}{\eta}\right)(1-l)(g \circ \nabla u)(t)
\end{aligned}
$$

Substuting (3.6) in (3.5), we get

$$
\begin{aligned}
\int_{\Omega} \nabla u(x, t) \cdot \int_{0}^{t} g(t-s) \nabla u(x, s) d s d x & \leq \frac{1}{2}\left(1+(1+\eta)(1-l)^{2}\right)\|\nabla u(., t)\|_{2}^{2} \\
& +\frac{1}{2}\left(1+\frac{1}{\eta}\right)(1-l)(g \circ \nabla u)(t) .
\end{aligned}
$$

Combining (3.3), (3.4) and (3.7), we find

$$
\begin{aligned}
\|\nabla u(., t)\|_{2}^{2} & \leq c_{1} \delta E(t)-\frac{C_{\delta}}{c_{0}} E^{\prime}(t) \\
& +\frac{1}{2}\left(1+(1+\eta)(1-l)^{2}\right)\|\nabla u(., t)\|_{2}^{2} \\
& +\frac{1}{2}\left(1+\frac{1}{\eta}\right)(1-l)(g \circ \nabla u)(t) .
\end{aligned}
$$

We then choose $0<\eta<l(2-l) /(1-l)^{2}$, which makes $c_{2}=\frac{1}{2}\left(1+(1+\eta)(1-l)^{2}\right)<1$, and, therefore, (3.8) takes the form

$$
\|\nabla u(., t)\|_{2}^{2} \leq c_{1} \delta E(t)-\frac{C_{\delta}}{c_{0}} E^{\prime}(t)+c_{2}\|\nabla u(., t)\|_{2}^{2}+c_{3}(g \circ \nabla u)(t),
$$

where $c_{3}=\frac{1}{2}\left(1+\frac{1}{\eta}\right)(1-l)$. This yields $(3.2)$ with $c_{4}=\frac{c_{1}}{1-c_{2}}$ and $c_{5}=\frac{c_{3}}{1-c_{2}}$.

Theorem 3.3. Let $u$ be the solution of (1.1). Then, there exist strictly two positive constants $\lambda_{0}$ and $\lambda_{1}$ such that the energy satisfies, for all $t \in \mathbb{R}^{+}$,

$$
\begin{gathered}
E(t) \leq \lambda_{0} e^{-\lambda_{1} \int_{0}^{t} \xi(s) d s \quad \text { if } p=1} \\
E(t) \leq \lambda_{0}\left(1+\int_{0}^{t} \xi^{2 p-1}(s) d s\right)^{\frac{-1}{2 p-2}} \quad \text { if } p>1 .
\end{gathered}
$$

Moreover, if $\xi$ and $p$ in $\left(G_{2}\right)$ satisfy

$$
\int_{0}^{+\infty}\left(1+\int_{0}^{t} \xi^{2 p-1}(s) d s\right)^{\frac{-1}{2 p-2}} d t<+\infty
$$

then, for all $t \in \mathbb{R}^{+}$,

$$
E(t) \leq \lambda_{0}\left(1+\int_{0}^{t} \xi^{p}(s) d s\right)^{\frac{-1}{p-1}} \quad \text { if } p>1
$$


Remark 3.1. Estimates (3.10) and (3.11) yield

$$
\int_{0}^{+\infty} E(t) d t<+\infty
$$

Proof. From (3.1) and for any $\kappa>0$, we have

$$
\begin{aligned}
E^{\prime}(t) & \leq 0=-\kappa E(t)+\kappa E(t) \\
& \leq-\kappa E(t)+\kappa\left(\frac{1}{2}(g \circ \nabla u)(t)+\frac{1}{2}\left(1-\int_{0}^{t} g(s) d s\right)\|\nabla u(., t)\|_{2}^{2}\right) \\
& \leq-\kappa E(t)+\frac{\kappa}{2}(g \circ \nabla u)(t)+\frac{\kappa}{2}\|\nabla u(., t)\|_{2}^{2} .
\end{aligned}
$$

Recalling Lemma 3.2, we get

$$
\begin{aligned}
E^{\prime}(t) \leq & -\kappa E(t)+\frac{\kappa}{2}(g \circ \nabla u)(t) \\
& +\frac{\kappa}{2}\left(c_{4} \delta E(t)-\frac{C_{\delta}}{c_{0}} E^{\prime}(t)+c_{5}(g \circ \nabla u)(t)\right) \\
\leq & -\kappa\left(1-\frac{c_{4}}{2} \delta\right) E(t)-\frac{\kappa C_{\delta}}{2 c_{0}} E^{\prime}(t)+\frac{\kappa\left(1+c_{5}\right)}{2}(g \circ \nabla u)(t) .
\end{aligned}
$$

Then we have

$$
\left(1+\frac{\kappa C_{\delta}}{2 c_{0}}\right) E^{\prime}(t) \leq-\kappa\left(1-\frac{c_{4}}{2} \delta\right) E(t)+\frac{\kappa\left(1+c_{5}\right)}{2}(g \circ \nabla u)(t) .
$$

By choosing $\delta$ small enough, we obtain, for two positive constants $\lambda$ and $\gamma$,

$$
E^{\prime}(t) \leq-\lambda E(t)+\gamma(g \circ \nabla u)(t) .
$$

Case of $p=1$. Multiplying (3.14) by $\xi(t)$ and exploiting $\left(G_{2}\right)$, we get

$$
\begin{aligned}
\xi(t) E^{\prime}(t) & \leq-\lambda \xi(t) E(t)+\gamma(\xi g \circ \nabla u)(t) \\
& \leq-\lambda \xi(t) E(t)-\gamma\left(g^{\prime} \circ \nabla u\right)(t) \\
& \leq-\lambda \xi(t) E(t)-\gamma E^{\prime}(t) .
\end{aligned}
$$

We then set $L=(\xi+\gamma) E \sim E$ to obtain, from (3.15) and the fact that $\xi^{\prime} \leq 0$,

$$
L^{\prime}(t) \leq-\lambda \xi(t) E(t) \leq-\lambda_{1} \xi(t) L(t) .
$$

A simple integration of (3.16) leads to

$$
L(t) \leq C e^{-\lambda_{1} \int_{0}^{t} \xi(s) d s} .
$$

This gives (3.9), by virtue of $L \sim E$.

Case of $p>1$. To establish (3.10), we again consider (3.14) and use Lemma 2.1 to get

$$
\xi(t) E^{\prime}(t) \leq-\lambda \xi(t) E(t)+C\left(-E^{\prime}(t)\right)^{\frac{1}{2 p-1}} .
$$


Multiplication of the last inequality by $\xi^{\alpha} E^{\alpha}(t)$, where $\alpha=2 p-2>0$, gives

$$
\frac{1}{\alpha+1} \xi^{\alpha+1} \frac{d}{d t} E^{\alpha+1}(t) \leq-\lambda \xi^{\alpha+1}(t) E^{\alpha+1}(t)+c(\xi E)^{\alpha}(t)\left(-E^{\prime}(t)\right)^{\frac{1}{\alpha+1}} .
$$

Use of Young's inequality, with $q=\alpha+1$ and $q^{*}=\frac{\alpha+1}{\alpha}$, yields, for any $\varepsilon>0$,

$$
\begin{aligned}
\frac{1}{\alpha+1} \xi^{\alpha+1} \frac{d}{d t} E^{\alpha+1}(t) & \leq-\lambda \xi^{\alpha+1}(t) E^{\alpha+1}(t)+C\left(\varepsilon \xi^{\alpha+1}(t) E^{\alpha+1}(t)-C_{\varepsilon} E^{\prime}(t)\right) \\
& =-(\lambda-\varepsilon C) \xi^{\alpha+1}(t) E^{\alpha+1}(t)-C_{\varepsilon} E^{\prime}(t)
\end{aligned}
$$

We then choose $0<\varepsilon<\frac{\lambda}{C}$ and recall that $\xi^{\prime} \leq 0$, to obtain, for $c_{6}>0$,

$$
\left(\xi^{\alpha+1} E^{\alpha+1}(t)\right)^{\prime}(t) \leq \xi^{\alpha+1} \frac{d}{d t} E^{\alpha+1}(t) \leq-c_{6} \xi^{\alpha+1}(t) E^{\alpha+1}(t)-C E^{\prime}(t)
$$

which implies

$$
\left(\xi^{\alpha+1} E^{\alpha+1}+C E\right)^{\prime}(t) \leq-c_{6} \xi^{\alpha+1}(t) E^{\alpha+1}(t) .
$$

Let $W=\xi^{\alpha+1} E^{\alpha+1}+C E \sim E$. Then

$$
W^{\prime}(t) \leq-C \xi^{\alpha+1}(t) W^{\alpha+1}(t)=-C \xi^{2 p-1}(t) W^{2 p-1}(t) .
$$

Integrating over $(0, t)$ and using the fact that $W \sim E$, we obtain, for some $\lambda_{0}>0$,

$$
E(t) \leq \lambda_{0}\left(\int_{0}^{t} \xi^{2 p-1}(s) d s+1\right)^{\frac{-1}{2 p-2}}
$$

so (3.10) holds

To establish (3.12), we put

$$
\eta(t)=\int_{0}^{t}\|\nabla u(t)-\nabla u(t-s)\|_{2}^{2} d s .
$$

Using Remark 3.1, we have

$$
\begin{aligned}
\eta(t) & \leq 2 \int_{0}^{t}\left(\|\nabla u(t)\|_{2}^{2}+\|\nabla u(t-s)\|_{2}^{2}\right) d s \\
& \leq \frac{4}{1-l} \int_{0}^{t}(E(t)+E(t-s)) d s \\
& =\frac{8}{1-l} \int_{0}^{t} E(s) d s<\frac{8}{1-l} \int_{0}^{+\infty} E(s) d s<+\infty .
\end{aligned}
$$

This implies that

$$
\sup _{t \in \mathbb{R}^{+}} \eta^{1-\frac{1}{p}}(t)<+\infty
$$


Assume that $\eta(t)>0$. Then, from (3.14), we find

$$
\begin{aligned}
\xi(t) E^{\prime}(t) & \leq-\lambda \xi(t) E(t)+\gamma \xi(t)(g \circ \nabla u)(t) \\
& =-\lambda \xi(t) E(t)+\gamma \frac{\eta(t)}{\eta(t)} \int_{0}^{t}\left(\xi^{p}(s) g^{p}(s)\right)^{\frac{1}{p}}\|\nabla u(t)-\nabla u(t-s)\|_{2}^{2} d s
\end{aligned}
$$

Applying Jensen's inequality (2.4) for the second term of the right-hand side of (3.18), with

$$
\Omega=[0, t], \quad f(s)=\xi^{p}(s) g^{p}(s) \quad \text { and } \quad h(s)=\|\nabla u(t)-\nabla u(t-s)\|_{2}^{2},
$$

to get

$$
\xi(t) E^{\prime}(t) \leq-\lambda \xi(t) E(t)+\gamma \eta(t)\left(\frac{1}{\eta(t)} \int_{0}^{t} \xi^{p}(s) g^{p}(s)\|\nabla u(t)-\nabla u(t-s)\|_{2}^{2} d s\right)^{\frac{1}{p}} .
$$

Therefore, using (3.17) we obtain

$$
\begin{aligned}
\xi(t) E^{\prime}(t) & \leq-\lambda \xi(t) E(t)+\gamma \eta^{1-\frac{1}{p}}(t)\left(\xi^{p-1}(0) \int_{0}^{t} \xi(s) g^{p}(s)\|\nabla u(t)-\nabla u(t-s)\|_{2}^{2} d s\right)^{\frac{1}{p}} \\
& \leq-\lambda \xi(t) E(t)+C\left(-g^{\prime} \circ \nabla u\right)^{\frac{1}{p}}(t)
\end{aligned}
$$

and then

$$
\xi(t) E^{\prime}(t) \leq-\lambda \xi(t) E(t)+C\left(-E^{\prime}(t)\right)^{\frac{1}{p}}
$$

If $\eta(t)=0$, then $s \rightarrow \nabla u(s)$ is a constant function on $[0, t]$. Therefore

$$
(g \circ \nabla u)(t)=0
$$

and hence we have, from (3.14),

$$
E^{\prime}(t) \leq-\lambda E(t)
$$

which implies (3.19).

Now, multiplying (3.19) by $\xi^{\alpha}(t) E^{\alpha}(t)$, for $\alpha=p-1$, and repeating the same computations as in above, we arrive at, for some $\lambda_{0}>0$,

$$
E(t) \leq \lambda_{0}\left(\int_{0}^{t} \xi^{p}(s) d s+1\right)^{\frac{-1}{p-1}}
$$

This completes the proof of our main result.

The following examples illustrate our result and show the optimal decay rate in the polynomial case: 
Example 3.1. Let $g(t)=a(1+t)^{-\nu}$, where $\nu>2$, and $a>0$ so that

$$
\int_{0}^{+\infty} g(t) d t<1
$$

We have

$$
g^{\prime}(t)=-a \nu(1+t)^{-\nu-1}=-b\left(a(1+t)^{-\nu}\right)^{\frac{\nu+1}{\nu}},
$$

where $b=\nu a^{-\frac{1}{\nu}}$. Then $\left(G_{2}\right)$ holds with $\xi(t)=b$ and $p=\frac{\nu+1}{\nu} \in\left(1, \frac{3}{2}\right)$. Therefore (3.11) yields

$$
\int_{0}^{+\infty}\left(b^{2 p-1} t+1\right)^{\frac{-1}{2 p-2}} d t<+\infty
$$

and hence, by (3.12), we get

$$
E(t) \leq C(1+t)^{\frac{-1}{p-1}}=C(1+t)^{-\nu},
$$

which is the optimal decay.

Example 3.2. Let $g(t)=a e^{-(1+t)^{\nu}}$, where $0<\nu \leq 1$, and $a>0$ is chosen so that (3.20) holds. Then

$$
g^{\prime}(t)=-a \nu(1+t)^{\nu-1} e^{-(1+t)^{\nu}} .
$$

Therefore $\left(G_{2}\right)$ holds with $p=1$ and $\xi(t)=\nu(1+t)^{\nu-1}$. Consequently, we can use (3.9) to deduce

$$
E(t) \leq C e^{-\lambda(1+t)^{\nu}}
$$

\section{Acknowledgment}

The authors thank KFUPM and Lorraine-Metz university for their continuous support. This work has been finalized during the visit of the third author to KFUPM in December 2016 and during the scholarship of the first author in Lorraine-Metz. This work has been partially funded by KFUPM under Project \# IP152-Math 212.

\section{References}

1. Berrimi S. and Messaoudi S.A., A decay result for a quasilinear parabolic system, Progress in Nonlinear Differential Equations and their Applications 53 (2005), 43-50.

2. Da Prato G. and Iannelli M., Existence and regularity for a class of integrodifferential equations of parabolic type, J. Math. Anal. Appl. 112 (1985), $36-55$.

3. Englern H., Kawohl B. and Luckhaus S., Gradient estimates for solutions of parabolic equations and systems, J. Math. Anal. Appl. 147 (1990), 309-329. 
4. Liu G. and Chen H., Global and blow-up of solutions for a quasilinear parabolic system with viscoelastic and source terms, Math. Methods Appl. Sci. 37 (2014), 148-156.

5. Messaoudi S.A. and Tellab B., A general decay result in a quasilinear parabolic system with viscoelastic term, Applied Mathematics Letters 25 (2012), 443-447.

6. Messaoudi S.A. and Al-Khulaifi W., General and optimal decay for a quasilinear viscoelastic equation, Applied Mathematics Letters 66 (2017), 16-22.

7. Nakao M. and Ohara Y., Gradient estimates for a quasilinear parabolic equation of the mean curvature type, J. Math. Soc. Japan 48 \# 3 (1996), 455-466.

8. Nakao M. and Chen C., Global existence and gradient estimates for the quasilinear parabolic equations of $m$-Laplacian type with a nonlinear convection term, Journal of Differential Equations 162 (2000), 224-250.

9. Nohel J.A., Nonlinear Volterra equations for the heat flow in materials with memory, Integral and functional differential equations, Lecture notes in Pure and Applied Mathematics, Marcel Dekker Inc. 1981.

10. Pucci P. and Serrin J., Asymptotic stability for nonlinear parabolic systems, Energy methods in continuum mechanics, Kluwer Acad. Publ., Dordrecht, 1996.

11. Yin H.M., On parabolic Volterra equations in several space dimensions, SIAM J. of Mathematical Analysis. 22 (1991), 1723-1737. 